\section{P1-326 100 YEARS OF SUICIDE IN BRIGHTON AND HOVE, ENGLAND}

doi:10.1136/jech.2011.142976f.18

${ }^{1,2} \mathrm{~T}$ Scanlon, ${ }^{* 1,3} \mathrm{~A}$ Memon, ${ }^{1} \mathrm{C}$ Dorling, ${ }^{4} \mathrm{~A}$ Walker. ${ }^{1} \mathrm{NHS}$ Brighton and Hove, Brighton, UK; ${ }^{2}$ Brighton and Hove City Council, Brighton, UK; ${ }^{3}$ Brighton and Sussex Medical School, Brighton, UK; ${ }^{4}$ Kent, Surrey and Sussex Deanery, London, UK

Introduction Compared with the suicide rates in England (12.0/ 100000 males, 3.7/100000 females), Brighton and Hove (B\&H) (population $=250000$ ) has the $3^{\text {rd }}$ highest rate in males (18.9/ $100000)$ and the highest rate in females $(10.2 / 100000)$. We investigated long-term trends in suicide by age, sex, and method in $\mathrm{B} \& \mathrm{H}$ from 1901 to 2008

Methods Age-standardised suicide rates (ASR) were calculated from 1901 to 2008. Information on suicides was obtained from the Reports of the Medical Officers/Directors of Public Health for B\&H and the Office of National Statistics.

Results Suicide rates in $\mathrm{B} \& \mathrm{H}$ were consistently higher than the rates in England for most of the $20^{\text {th }}$ Century. The male:female ratio fluctuated from 3:1 in the 1920 s to $1: 1$ in 1960s to $2: 1$ in 2000 s. The ASR (per 100000 ) in males fluctuated from 31.2 in the 1920 s to 16.3 in $1960 \mathrm{~s}$ to 27.0 in $2000 \mathrm{~s}$, and from 11.0 in the $1920 \mathrm{~s}$ to 16.5 in $1960 \mathrm{~s}$ to 11.6 in 2000s in females. Coal gas inhalation was the most common method in both sexes ( $22 \%$ males, $41 \%$ females) in the 1920 s. This was replaced by self-poisoning in 1960s (39\% males, $57 \%$ females). In the 2000s, hanging became more common in males (37\%), whereas self-poisoning remained the most common method in females (45\%).

Conclusion The epidemiology of suicide in $\mathrm{B} \& \mathrm{H}$ has varied over the past 100 years. However, in contrast with the national decline in suicide rates, $\mathrm{B} \& \mathrm{H}$ rates have consistently remained high. These finding are discussed in light of information obtained from the local Public Health Reports/suicide audit.

\section{P1-327 EVIDENCE SUPPORTING THE USE OF REDUCED DOSE SCHEDULES FOR PNEUMOCOCCAL CONJUGATE VACCINES: SYSTEMATIC REVIEW AND META-ANALYSIS}

\author{
doi:10.1136/jech.2011.142976f.19
}

P Scott, ${ }^{*}$ L Bermetz, N Robert, N Low. Institute of Social and Preventive Medicine (ISPM), University of Bern, Bern, Switzerland

Introduction Pneumococcal conjugate vaccines (PCVs) are designed to protect against Streptococcus pneumoniae disease. The first PCV (7valent) was licensed based on clinical efficacy of 3 primary doses in infancy and a booster (" $3 p+1$ "). Many countries have since introduced reduced dose schedules; in 2010, 19 countries were using $2 p$ +1 and 21 were using $3 p+1$ schedules. Evidence supporting the use of the $2 p+1$ schedule was examined in a systematic review.

Methods We searched 12 databases up to March 2010. We included randomised controlled trials (RCT) and case-control studies comparing $2 p+1$ to $3 p+0$ or $3 p+1$ schedules. Data on clinical outcomes, nasopharyngeal carriage and seropositivity (ELISA antibody concentration $>0.35 \mu \mathrm{g} / \mathrm{ml}$ for all studies and serotypes) or geometric mean concentrations (GMC) were analysed.

Results There were no RCTs reporting clinical or carriage outcomes for direct comparisons between $2 p+1$ and $3 p+0$ or $3 p+1$ schedules. Percentages seropositive, or GMC following $2 p+1$ and $3 p+1$ schedules ( 3 RCTs) were similar for most serotypes, except $6 \mathrm{~B}$ and $23 \mathrm{~F}$, where $3 p+1$ schedules were consistently favoured. GMC (1 RCT) at 13 months were substantially higher in the $2 p+1$ than the $3 p+0$ group. Differences were smaller 6 months later.

Conclusion Clinical and carriage data are absent for direct comparisons (RCTs) between $2 p+1$ and $3 p+0$ or $3 p+1$ schedules. Evidence supporting a $2 p+1$ schedule is based on limited RCT data about immunogenicity (where the correlation with protection against clinical disease is not well defined), non-randomised comparisons, or indirect comparisons of post-introduction surveillance data.

\section{P1-328 COMPARISON OF PREVENTIVE PRACTICES AMONG WOMEN IN SÃO PAULO/BRAZIL}

doi:10.1136/jech.2011.142976f.20

${ }^{1} \mathrm{~N}$ Segri, ${ }^{3} \mathrm{P}$ Francisco, ${ }^{5} \mathrm{C}$ Alves, ${ }^{3} \mathrm{M}$ Barros, ${ }^{1} \mathrm{C}$ Cesar, ${ }^{2} \mathrm{M}$ Goldbaum, ${ }^{4} \mathrm{D}$ Malta ${ }^{1}$ Faculty of Public Health, USP, São Paulo, Brazil; '2University of São Paulo, São Paulo, Brazil; ${ }^{3}$ University of Campinas, Campinas, São Paulo, Brazil; ${ }^{4}$ Brazilian Ministry of Health, Brasilia, Distrito Federal, Brazil, ${ }^{5}$ State Health Institute, São Paulo, Brazil

The aim of this study was to compare estimates of Pap smear screening and mammogram obtained from household survey and a telephone survey in São Paulo/Brazil in the year 2008, according to sociodemographic characteristics, and to dimension the observed differences from those estimates. The study used data from the ISACapital 2008 Survey, conducted in São Paulo city, by Faculty of Public Health of the University of São Paulo with support from the Municipal Health Department, and VIGITEL (São Paulo), a telephone survey conducted by the Brazilian Ministry of Health towards Surveillance of Risk and Protection Factors for Chronic non-transmittable Diseases. Estimates of Pap smear and mammogram in women, as well as its realisation in the year prior to the interview were compared according to type of survey (household/ telephone) by Poisson regression adjusted for age and education. There were no statistically significant differences between the estimates obtained by VIGITEL and ISA-Capital for the prevalence of mammogram in the year prior to the interview. However, estimates for the global estimates of the Pap smear at least once in life and in the last year and mammogram at least once in life, there has been observed statistically significant differences, with higher prevalence among those interviewed by telephone. It shows a tendency to overestimate the prevalence of mammography and Pap smear in the telephone survey data indicating a necessity for new studies that also contribute to a better understanding of the differences observed using different types of surveys.

\section{P1-329 SOCIOECONOMIC INEQUALITIES IN CANCER RISK BY SITE, AGE, AND SEX IN SCOTLAND, 2000-2007}

doi:10.1136/jech.2011.142976f.21

${ }^{1} \mathrm{~K}$ Sharpe, ${ }^{* 1,2} \mathrm{~A}$ McMahon, ${ }^{1} \mathrm{P}$ McClements, ${ }^{1,3} \mathrm{D}$ Brewster, ${ }^{1,2} \mathrm{D}$ Conway. ${ }^{1}$ Information Services Division, NHS National Services Scotland, Edinburgh, UK; ${ }^{2}$ University of Glasgow, College of Medical, Veterinary and Life Sciences: Dental School, Glasgow, UK; ${ }^{3}$ Public Health Sciences, Edinburgh University Medical School, Edinburgh, UK

Some cancers are associated with socioeconomic inequality (SEI); this study quantified the extent and differences by cancer type, age and sex. We reviewed 216315 incident cancers (excluding non melanoma skin cancer) from 2000 to 2007 classified into 27 anatomical groups. Further analyses were performed by morphology or sub site. Deprivation was measured using the Scottish Index of Multiple Deprivation and SEI using the slope index of inequality and the relative index of inequality (RII). Analyses were partitioned by five-year age group and sex. For both sexes, incidence was positively associated with deprivation for lung, head and neck, stomach, oesophagus, bladder, liver, pancreas and negatively associated with deprivation for cutaneous melanoma. Prostate, rectum (male), cervical and breast (female) cancers also show inequalities; only prostate and breast cancers are negatively associated with deprivation. Female RII (0.36) was lower than male RII (0.53). For males, SEI is pronounced at ages $45-74$ years, peaking at 60-64 years 
$(\mathrm{RII}=0.39-0.58)$. For females, SEI begins at $20-24$ years $(\mathrm{RII}=0.27)$ with pronounced inequalities at 60-79years and peaking at 65-69 years $(\mathrm{RII}=0.30-0.48)$. All four morphology groups demonstrate inequalities for lung cancer. For cervical cancer, squamous cell carcinoma dominates; in oesophageal cancer, squamous cell carcinoma followed by adenocarcinoma and ultimately other morphologies show inequalities. For head and neck cancers; hypopharynx, piriform sinus and larynx followed by lip, oral cavity and ultimately oropharynx, base of tongue, palate and tonsil show inequalities. We conclude: age, morphology, sex and site provide important information to better understand SEI.

\section{P1-330 INEQUALITIES IN DENTAL CARIES: THE ROLE OF NMES INTAKE}

doi:10.1136/jech.2011.142976f.22

${ }^{1}$ A Sherriff, ${ }^{1} \mathrm{M}$ Wilson, ${ }^{2} \mathrm{~J}$ Armstrong, ${ }^{2} \mathrm{Y}$ Brogan, ${ }^{1} \mathrm{~L}$ Macpherson. ${ }^{1}$ Glasgow University, Glasgow, UK; ${ }^{2}$ Glasgow Caledonian University, Glasgow, UK

Introduction Dental caries is one of the most prevalent diseases of childhood in the UK, with a disproportionate burden experienced by the most disadvantaged groups. Non-milk extrinsic sugar (NMES) intake, poor oral hygiene and acidogenic bacteria are considered the main risk factors for caries, however, their role in explaining the observed inequalities has not been fully explored. The aim of the study is to assess the extent to which these factors explain the socio-economic (SES) inequalities in caries.

Methods Data on treatment for decay (caries), SES (Scottish Index of Multiple Deprivation (SIMD)), NMES intake and oral hygiene was obtained from 1491 children participating in the Survey of Sugar Intake among Children in Scotland (3-17 years). Logistic regression models assessed the impact on the Caries-SES relationship of NMES intake and oral hygiene. A priori interactions tests were performed.

Results $54 \%$ of children had caries and there was a strong SES gradient $(p<0.001)$. The OR $[95 \% \mathrm{CI}]$ for caries in the most deprived vs least deprived groups was 3.9 [2.8 to 5.5], and increased slightly when adjustments were made for NMES intake and oral hygiene (AOR $[95 \% \mathrm{CI}]=4.3$ [2.9 to 6.3]). There was no evidence of an interaction between NMES intake/Oral hygiene, SES and caries $(\mathrm{p}=0.4 ; \mathrm{p}=0.7)$.

Conclusions The SES patterning of caries is not attenuated by NMES intake and/or oral hygiene. Further work is required to explore alternative pathways to explaining the observed inequalities in oral health and may focus on the interaction between diet and acidogenic bacteria.

\section{P1-331 OUTCOMES OF SCREENING MAMMOGRAPHIES IN ONE COMMUNITY-BASED INITIATIVE IN LEBANON}

doi:10.1136/jech.2011.142976f.23

J Sidaoui, ${ }^{*}$ S Adib. University of St Joseph, Beirut, Lebanon

Introduction and objectives There is no formal breast screening program currently in Lebanon. This report describes the outcomes of a screening mammography initiative implemented by one Lebanese non-governmental organization. The objectives were to assess the socio-demographic and reproductive characteristics associated with repeating a screening mammography after receiving reassuring results from a first one, and to estimate, for the first time in Lebanon, the proportion of mammographies suggesting malignancy which could be obtained from a mass screening activity.
Methods A total of 1500 women participated in the "Faire Face" screening initiative between 2002 and 2009, which allowed them to obtain repeated annual mammographies free of charge.

Results Of participants, 58\% repeated the test at least once even after receiving reassuring results. Factors associated with test repetition were older age, fewer children and retirement but not housewife or employed status. Results were suspicious (ACR4 or 5) in $2.4 \%$ of cases.

Discussion and conclusions The proportion of suspicious readings on mammograms which require further investigation was similar to European figures. Evidence shows that, given the opportunity, increasingly more women of younger ages are willing to undergo the required annual test, and to repeat it. Several issues have to be debated nationally including lowering the financial barriers to mammography for all women regardless of their socio-demographic backgrounds, and improving the opening hours of mammography test centers to attract working women or those with larger families, and therefore with limited free time in the morning.

\section{P1-332 FIRST INSIGHT INTO GENETIC DIVERSITY OF MYCOBACTERIUM TUBERCULOSIS STRAINS IN SALVADOR, BRAZIL}

doi:10.1136/jech.2011.142976f.24

${ }^{1}$ A Silva, ${ }^{*}{ }^{3}$ L Ferrazoli, ${ }^{1} \mathrm{~J}$ Reis, ${ }^{2} \mathrm{~S}$ Pereira, ${ }^{2} \mathrm{E}$ Mota, ${ }^{1} \mathrm{M}$ Reis. ${ }^{1}$ Centro de pesquisa gonçalo muniz, Fundação oswaldo cruz-bahia, Salvador, Bahia, Brazil; ${ }^{2}$ Universidade federal da Bahia, Instituto de saúde coletiva, Salvador, Bahia, Brazil; ${ }^{3}$ Instituto adolfo lutz, IAL, São Paulo, São Paulo, Brazil

This study constitutes a first attempt to describe the genetic population structure of $M$ tuberculosis circulating in Salvador. A total of 56 confirmed cases of pulmonary tuberculosis, identified between March and June, 2008 were analysed using IS6110-restriction fragment length polymorphism (RFLP). The study population was characterised by male (71.4\%) and over 30 years of age $(68.7 \%)$. Forty-one isolates were found in a single pattern (73.21\%), while 15 $(26.78 \%)$ were found in group patterns, forming six clusters. The higher rate of diversity observed is much more suggestive of endogenous reactivation than recent transmission.

\section{P1-333 YEARS OF POTENTIAL LIFE LOST FROM ASSAULT WITH FIREARMS OR SHARP OBJECTS AMONG ADOLESCENTS IN CAMPO GRANDE, MS, BRAZIL, IN 2007}

doi:10.1136/jech.2011.142976f.25

A P Silva, ${ }^{*}$ E R Pontes, J R Tognini. Universidade Federal de Mato Grosso do Sul, Campo Grande, Brazil

Introduction In Brazil, mortality by external causes has reached epidemic proportions, having become the principal cause of death among male adolescents (Brazil, 2010). The aim of this study was to evaluate the years of potential life lost by this adolescent group in Campo Grande, MS, Brazil, in 2007.

Methods This retrospective study investigated deaths caused by assault with firearm discharge or sharp objects, as described in Chapter XX of the ICD-10 (WHO, 1996). The proportion of these deaths among adolescents aged 15 to 19 years was calculated. Data were collected from the Datasus database of the Brazilian Unified Health Care System.

Results For every 1000 deaths occurring in this age range in 2007, 528 were caused by assault with firearms or sharp objects. Considering that life expectancy in Mato Grosso do Sul State is 73.8 years (IBGE, 2007), 58.8 years of potential life are lost by every 15 -year-old victim. 